\title{
CARACTERIZACIÓN DE TRAUMA POR ACCIDENTE EN MOTOCICLETA, PACIENTES DE HOSPITALDE LEMPIRA. PRIMER SEMESTRE 2016.
}

\author{
Characterization of trauma by accident in motorcycles, \\ Patients from Hospital of Lempira at first. \\ *Gerardo José Ayestas Moreno, *Gabriel Adalberto Pérez Lara.
}

\section{RESUMEN}

Cada año muchos hondureños mueren a causa de accidentes por motocicleta. En el 2015 fallecieron 259 personas a nivel nacional. Objetivo: Describir las características clínicas-traumatológicas y región anatómica con mayores lesiones provocadas por trauma debido a accidente en motocicleta, pacientes adultos del Hospital Juan Manuel Gálvez en el primer semestre de 2016. Pacientes y métodos: Estudio descriptivo, de corte transversal, se aplicó instrumento de recolección de datos (cuestionario) a pacientes hospitalizados por trauma por motocicleta de enero a junio de 2016; la población/muestra de 59 pacientes. Resultados: $50(84.7 \%)$ casos fueron hombres y 9 $(15.3 \%)$ mujeres, con edad promedio de 29.6 años. $16(27 \%)$ pacientes afirmó haber consumido alcohol previo al accidente. Se manejó quirúrgicamente 21 (35\%) pacientes, las cirugías ortopédicas representaron $87 \%$. El promedio de hospitalización de los pacientes quirúrgicos fue mayor de 4 días. La mortalidad fueron 2 casos (3.4\%), provocado por trauma cráneo encefálico (TEC). Los tipos de traumas fueron: osteomuscular $(37.3 \%)$, TEC (34\%), politraumatismo (13.5\%), contusiones $(6.8 \%)$, traumas faciales $(3.4 \%)$ y traumas torácicos $(5 \%)$. Conclusiones: Los hombres en edad reproductivas representaron el mayor número de casos. La región anatómica más lesionada fue osteomuscular, sin embargo la mortalidad fue mínima. Las lesiones osteomusculares ameritaron manejo quirúrgicos en su mayoría. Siendo un problema de importancia socioeconómica y de salud local.

*Doctor en medicina y cirugía, Egresado la Universidad Nacional autónoma de Honduras en el Valle de Sula.

Dirigir correspondencia a: gerardo3_7@hotmail.com

Recibido: 19 de marzo $2017 \quad$ Aprobado: 04 de septiembre 2017

\section{PALABRAS CLAVE}

Accidente de tránsito, hospitalización, motocicletas, trauma.

\section{ABSTRACT}

Each year many Hondurans die of accidents by motorcycle. 259 people nationally died in 2015. Objective: Describe the clinicas-traumatologicas characteristics and anatomical region with major injuries caused by accident in motorcycle, in adult patients of the Hospital Juan Manuel Galvez in the first half of 2016. Patients and methods: descriptive, crosssectional study, collecting data (questionnaire) instrument was applied to patients hospitalized for trauma by motorcycle from January to June 2016; population/sample of 59 patients. Results: $50(84.7 \%)$ of the cases were men and $9(15.3 \%)$ women, with an average age of 29.6 years; $16(27 \%)$ patients claimed to have consumed alcohol prior to the accident. Was managed surgically $21(35 \%)$ patients, orthopaedic surgeries accounted for $87 \%$. The patients surgical was more than 4 days of hospitalization. Mortality was 2 cases (3.4\%), trauma-induced head injury (TEC). Types of traumas were: Musculoskeletal (37.3\%), TEC (34\%), polytraumatism $(13.5 \%)$, contusions $(6.8 \%)$, trauma facial $(3.4 \%)$ and trauma thoracic (5\%) Conclusions: Men of reproductive age acco-unted for the largest number of cases. The most injured anatomical region was osteomuscular, however, mortality was minimal. Musculoskeletal injuries warranted surgical management for the most part. Being a problem of socioeconomic importance and local health.

\section{KEYWORDS}

Accidents Traffic, hospitalization, motorcycles, trauma. 


\section{INTRODUCCIÓN}

Para conducir una motocicleta con prudencia en el tráfico es necesario contar con ciertas aptitudes y conocimientos. Lo que el conductor haga antes de iniciar un viaje será clave para determinar si efectivamente llegará o no al lugar de destino sin riesgos. Por norma, antes de iniciar cualquier viaje, un conductor prudente tiene que: 1. Utilizar el equipo adecuado. 2. Familiarizarse con la motocicleta. 3. Controlar el equipo de la motocicleta. 4. Ser un conductor responsable. ${ }^{(1)}$

Honduras representa un país en desarrollo en donde en los últimos 5 años se ha visto un creciente aumento del parque vehicular de motocicletas en un $150 \%$, ${ }^{(2)}$ el riesgo a accidentes de tránsito que representan estos vehículos que son de fácil acceso a la población, radica en el peligro y vulnerabilidad física que las personas tienen. Es importante identificar factores de riesgo/ mórbidos que influyen en provocar traumas por motocicleta y su relación con el tipo de lesión, esto va ligado con la edad del conductor, el género, en el caso de estudio, la obediencia en el uso del casco es determinante en el desenlace del accidente. ${ }^{(3-5)}$

En el 2014 en Honduras, la Dirección Nacional de Tránsito informó que ocurrieron 1097 accidentes por motocicleta a nivel nacional, muriendo 204 personas. ${ }^{(6)}$

Aunque las lesiones afectan a las personas en todas las sociedades, independientemente de su nivel de desarrollo, las sociedades de los países en desarrollo se encuentran en mayor peligro y son afectadas desproporcionadamente por las consecuencias de las lesiones. ${ }^{(7)}$

Estimar los costos de los choques y los traumatismos causados por el tránsito puede ayudar a los países a comprender la gravedad del problema que plantean y las ventajas de invertir en medidas para prevenirlos, así como la obediencia en el uso del casco el cual es determinante en el desenlace del accidente, es decir la fatalidad del mismo. ${ }^{(8)}$
Año tras año, la mortalidad relacionada con esta causa cobra muchas vidas, además conlleva muchas incapacidades psicofísicas que producen un impacto importante a nivel de la salud pública en la población. ${ }^{(9)}$

El presente estudio, se realizó con el propósito de describir las características clínicas-traumatológicas y región anatómica con mayores lesiones provocadas por accidente en motocicleta, en pacientes adultos del Hospital Juan Manuel Gálvez en el primer semestre de 2016.

\section{PACIENTES Y MÉTODOS}

Estudio con enfoque cuantitativo, alcance descriptivo y corte transversal, realizado en pacientes hospitalizados por lesiones causadas por motocicletas, en el Hospital Juan Manuel Gálvez, de enero a junio de 2016.

El universo/ muestra, fueron 59 pacientes con trauma por accidente de motocicleta que ingresaron a la emergencia del Hospital Juan Manuel Gálvez (HJMG) en el período descrito. (Tipo conductor o pasajero).

Para la recolección de datos, se aplicó un instrumento tipo cuestionario, que fue llenado con datos del expediente clínico.

Tabulación y análisis de datos mediante programa SPSS, versión 22.

\section{Criterios de Inclusión:}

Pacientes adultos (mayores de 18 años), de ambos sexos, con historia de accidente en motocicleta, transportados primariamente en calidad de conductor o pasajero, que fueron hospitalizados y recibieron atención médica en HJMG de enero a junio 2016, se incluyeron también los pacientes con trauma antiguo, con secuelas o complicaciones ingresados en dicha fecha.

\section{Criterios de Exclusión:}

Pacientes que asistieron por lesiones asociadas a automóvil, bicicleta u otro trasporte, que recibieron lesiones por motocicleta de manera externa y/o circunstancial. Se exclu- 
yeron también los pacientes fuera de los límites de fecha predeterminados, los pacientes de edad pediátrica (0-18 años) pacientes con cuyos datos clínicos no estaban completos en el expediente.

\section{RESULTADOS}

Los pacientes entre 18-25 años conformaron el $49 \%$ total de los caso, con una edad promedio de los accidentados de 29.6 año. En relación al sexo de los accidentados fue $50(84.4 \%)$ hombres y $9(15.3 \%)$ mujeres. (Ver Tabla No. 1).

Tabla No. 1: Distribución por sexo y edad de los pacientes.

\begin{tabular}{|c|c|c|c|c|c|}
\hline \multirow{2}{*}{ SEXO } & \multicolumn{4}{|c|}{ EDAD } & \multirow{2}{*}{} \\
\cline { 2 - 5 } & $18-25$ & $26-32$ & $33-56$ & $57-76$ & Total \\
\hline Mujer & 3 & 5 & 1 & 0 & $\begin{array}{c}9 \\
(15.3 \%)\end{array}$ \\
\hline Hombre & 26 & 14 & 8 & 2 & $\begin{array}{c}50 \\
(84.7 \%)\end{array}$ \\
\hline Total & $\begin{array}{c}29 \\
(49.1 \\
\%)\end{array}$ & $\begin{array}{c}19 \\
(32.2 \\
\%)\end{array}$ & $\begin{array}{c}(15.3 \\
\%)\end{array}$ & $\begin{array}{c}2 \\
(3.4 \\
\%)\end{array}$ & $\begin{array}{c}59 \\
(100 \%)\end{array}$ \\
\hline
\end{tabular}

Fuente: Instrumento de recolección de datos

Del total de pacientes, 38 (64\%) fueron debido a colisión, 17 (29\%) debido a choque y en $4(7 \%)$ fueron otros mecanismos. (Ver Gráfico No.1).

Los pacientes que utilizaban casco al momento del accidente fueron 37 casos (63\%), mientras los que no lo utilizaban fueron 13 casos $(22 \%)$ y en los demás casos se desconoce $9(15 \%)$.

Alcoholismo: Según los datos encontrados sobre los pacientes accidentados 66\% (39 casos) refirieron que no habian consumido alcohol. (Ver Gráfica No. 1).
Gráfica No. 1: Pacientes que refirieron consumo de alcohol previo al accidente.

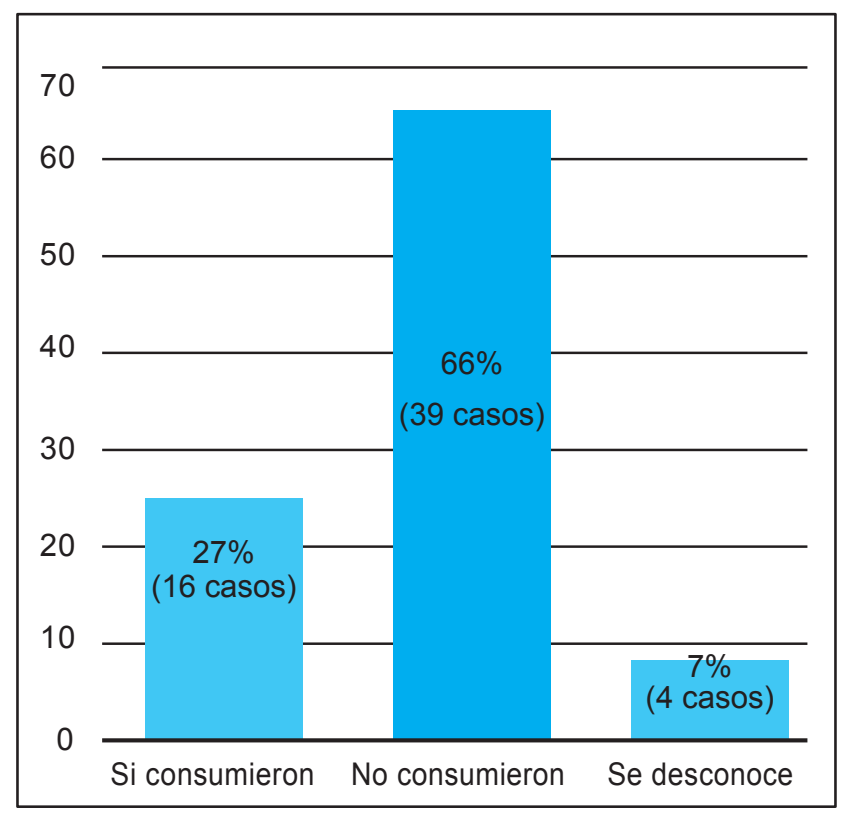

Fuente: Instrumento de recolección de datos

A 34 casos $(57.6 \%)$ de los pacientes accidentados se les dio un manejo conservador. (Ver Gráfica No. 2).

Gráfica No. 2: Conducta terapéutica realizada.

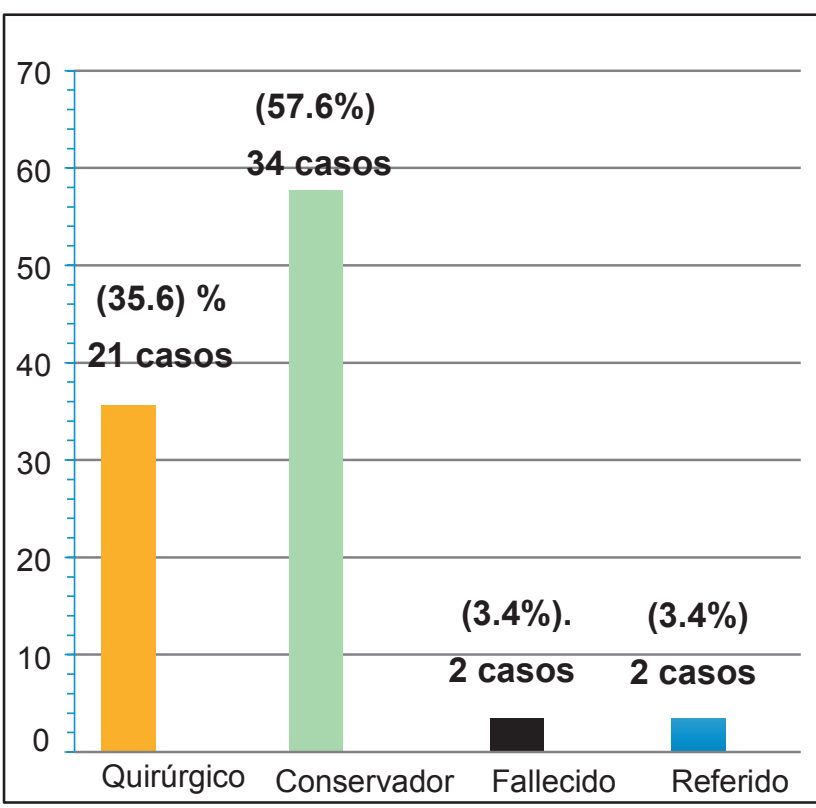

Fuente: Instrumento de recolección de datos

El manejo quirúrgico representó un 21 $(35.6 \%)$ de todos los pacientes: $18(87 \%)$ 
fueron cirugías ortopédicas, y $3(13 \%)$ fueron cirugías de abdomen, tórax y otras. La mortalidad ocurrió en 2 casos ( $3.4 \%$ ), en pacientes del sexo masculino, la causa fue por trauma cráneoencefálico, asociado al no uso de casco, y ocurrio en los primeros minutos de su llegada al hospital.

El trauma osteomuscular representó (22) $37.2 \%$ de los accidentes. El TEC ocurrió en (20) $34 \%$ de los casos, de estos: 18 presentaron TEC leve (Glasgow 14-15) y 2 sufrieron TEC moderado, de los cuales 1 presentó Glasgow de 10 y otro 12. Al momento del ingreso 3 pacientes $(5 \%)$ no portaban casco y tenían alteración de su estado de conciencia. (Ver Gráfica No. 3).

Gráfica No. 3: Tipo de trauma presentaron los pacientes.

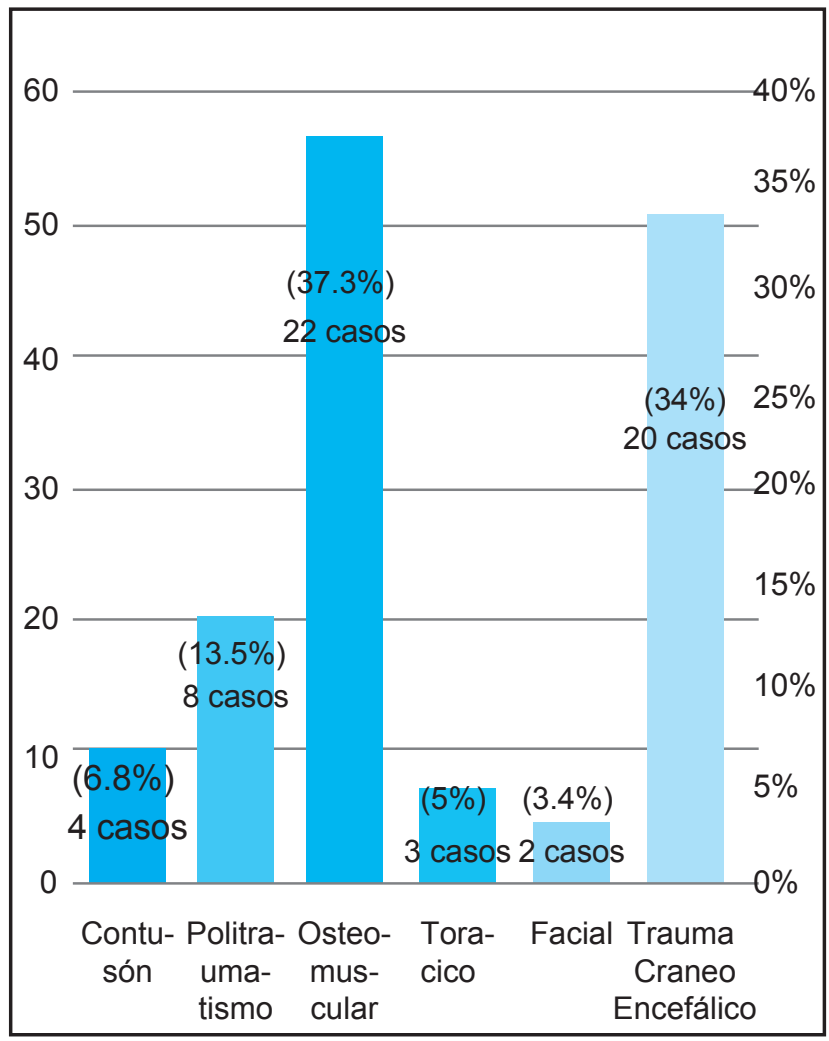

Fuente: Instrumento de recolección de datos

La moda de tiempo de hospitalización fue de 2 días en un $35.6 \%$ (21 casos). El tiempo de Hospitalización de los pacientes manejados quirúrgicamente fue de 4 días en un $13.5 \%$ (8 casos). (Ver Tabla No. 2).
Tabla No. 2: Relación de los manejos y días de hospitalización.

\begin{tabular}{|c|c|c|c|c|c|c|}
\hline Días & $\begin{array}{c}\text { Manejo } \\
\text { Clínico }\end{array}$ & $\begin{array}{c}\text { Manejo } \\
\text { Quirúr- } \\
\text { gico }\end{array}$ & $\begin{array}{c}\text { Falle- } \\
\text { cidos }\end{array}$ & $\begin{array}{c}\text { Refe- } \\
\text { ridos }\end{array}$ & \multicolumn{2}{|c|}{ Total } \\
\hline $\mathbf{1}$ & 12 & 1 & 2 & 1 & 16 & $27.1 \%$ \\
\hline $\mathbf{2}$ & 16 & 5 & 0 & 0 & 21 & $35.5 \%$ \\
\hline $\mathbf{3}$ & 4 & 2 & 0 & 1 & 7 & $11.8 \%$ \\
\hline $\mathbf{4}$ & 1 & 8 & 0 & 0 & 9 & $15.2 \%$ \\
\hline $\mathbf{5}$ & 0 & 2 & 0 & 0 & 2 & $3.3 \%$ \\
\hline $\mathbf{6}$ & 0 & 1 & 0 & 0 & 1 & $1.5 \%$ \\
\hline $\mathbf{7}$ & 0 & 2 & 0 & 0 & 2 & $3.3 \%$ \\
\hline $\mathbf{8}$ & 1 & 0 & 0 & 0 & 1 & $1.5 \%$ \\
\hline Total & $\mathbf{3 4}$ & $\mathbf{2 1}$ & $\mathbf{2}$ & $\mathbf{2}$ & $\mathbf{5 9}$ & $\mathbf{1 0 0} \%$ \\
\hline
\end{tabular}

Fuente: Instrumento de recolección de datos

\section{DISCUSIÓN}

En este estudio, las personas del sexo masculino conformaron el $84.7 \%$ de los casos y la edad reproductiva entre 18-25 años y fueron los que más sufrieron accidentes por motocicleta en un $49.1 \%$ de los casos, dándose una edad promedio de 29.1 años de los pacientes del estudio lo que difiere con estudios de perfil europeo en donde edad promedio total es mayor (35 años) hasta en un $50 \% .{ }^{(10)}$

Los hombres jóvenes tienen 2 veces más probabilidades de morir o sufrir traumatismos en los accidentes de tránsito que las mujeres, señala el estudio de siniestralidad de Madrid del 2013.(11) El presente estudio de relevancia local ratifica la elevada incidencia accidentes en las personas del sexo masculino $(84.7 \%)$ que más sufrieron accidentes por motocicleta, junto con la mortalidad del estudio que fue de $3.4 \%$ ( 2 casos), también de personas del sexo masculino.

En relación al uso del casco, fue afirmativo en $64 \%$ (37 casos) sin embargo los que no lo utilizaban fueron $13(22 \%)$ de los casos; dentro de este grupo estaban los tres pacientes con glasgow de 14 o menos. Siendo un factor prevenible, reafirmando el estudio de Donate, donde el no uso de casco aumenta en un $50 \%$ el riesgo de daño encefálico severo al momento de un accidente por motocicleta. ${ }^{(12)}$ 
Existe una serie de factores que incrementan la posibilidad de ser víctima de un accidente de tránsito, entre ellos destaca el conducir bajo la influencia del alcohol y la velocidad, como señala el estudio peruano en Heredia y Donate, donde este factor estuvo ligado hasta en un $50 \% .^{(7,12)}$ Sin embargo en este estudio, 16 pacientes (27\%) estuvieron ligados al factor alcohólico; y el tipo de accidente que presentaron los pacientes fue tipo colisión en un $64 \%$ de los casos, lo que nos indica que el conductor iba en movimiento, pudiendo asociarse a exceso de velocidad como lo reportado por estudios previos y factores de riesgo como los mencionados por el departamento de ingeniería vial en Colombia en 2013,(13) donde se encontró que el factor humano representó un $90 \%$ de las causas de estos accidentes.

Los traumas osteomusculares de miembros superior e inferior representaron $37.3 \%$ de los casos, y en segundo lugar encontramos el trauma cráneoencefálico que representó $34 \%$ de las lesiones sufridas por los pacientes y en menor porcentaje, se presentaron los traumas faciales $3.4 \%$ y torácicos $5 \%$, resultando sumamente distinto con las estadísticas del Instituto Colombiano de Medicina Legal y Ciencias Forenses el cual en el 2009 encontró que $83 \%$ de las víctimas sufren lesiones de tipo craneoencefálico; $39 \%$ fracturas y mutilaciones de extremidades superiores e inferiores y $36 \%$ fracturas del esternón y costillas, ${ }^{(14)}$ estos resultados fueron distintos en relación a otros investigados y representaron mayor costo hospitalario por volumen y tratamientos que representan las secuelas en el paciente con trauma osteomuscular. ${ }^{(3)}$

El índice de mortalidad del estudio fue de $3.4 \%$ de los casos (2 pacientes), debido a TEC; un resultado sumamente menor, en comparación con la mortalidad observada en áreas urbanas del país (San Pedro Sula y Tegucigalpa) donde la cifra de mortalidad por accidentes de motocicleta alcanzo $10 \%$ en el año 2015 (2) y de estudios internacionales realizados en áreas urbanas donde la mortalidad alcanza hasta el $9.2 \%$ de casos. ${ }^{(15)}$

Conclusiones, los pacientes que presentan mayor incidencia en estos accidentes son del sexo masculino en edad reproductiva siendo un problema de importancia socioeconomica y de salud local, en el cual los factores exógenos (velocidad, consumo de alcohol) tuvieron relevancia importante en estos tipos de lesiones. Se demuestra que el no uso del casco aumenta el riesgo de mortalidad ya que los 2 casos de pacientes fallecidos del estudio fueron asociados a esta causa.

Las lesiones más frecuentes fueron los traumas osteomusculares de miembros superiores e inferiores y traumatismo craneoencefálico, ameritando mayor tiempo de estadía hospitalaria.

Recomendación, se recomienda la socialización mediática así como educación vial a la población local sobre factores prevenibles (no uso de casco, consumo de alcohol, exceso de velocidad) que causan y agravan los traumas por motocicleta. También capacitar al personal de salud ayudándose del valor descriptivo de los estudios de trauma para así mejorar el manejo hospitalario y reducir la mortalidad y complicaciones de estos casos.

\section{AGRADECIMIENTO}

Al personal Administrativo y Clínico del Hospital Juan Manuel Gálvez por facilitar apoyo logístico y acceso a expediente clínico y otros registros clínicos oficiales.

\section{REFERENCIAS BIBLIOGRÁFICAS}

1. Motorcycle Safety Foundation (MSF). Manual para usuarios de motocicletas. California: MSF; (s.f.) [Citado 15 de febrero de 2016.], disponible en:https:// dmv. dc.gov/sites/default/files/dc/sites/dmv/pu blication/attachments/mom_Spa nish.pdf.

2. La Tribuna. 21 personas mueren cada 
mes por accidentes en motocicletas. [internet] La tribuna, Honduras: 2016, febrero15. [Citado 15 de febrero de 2016.], Disponible en: http://www.latribuna.hn/ 2016/02/15/21-personas-mueren-cada -mes-por-accidentes-en-motocicle tas/.

3. Nantulya VM, Sleet DA, Reich MR, Rosenberg M, Peden M, Waxweiler R. The global challenge of road traffic injuries: can we achieve equity in safety? Inj Control Saf Promot. 2003;10(1-2):3-7.

4. Organización Mundial de la Salud. Informe Anual sobre Seguridad Vial. [internet]2009 [citado en junio de 2009].

Disponible en: http://www.who.int/violen ce_injury_prevention/road_safety_sta tus/2009/es/.

5. Nantulya VM. Equity dimensions of road traffic injuries in low and middle-income countries. Injury Control and Safety Promotion; 2010. 10 (7): 13-20.

6. El Heraldo. El 2015 ya registra 80 muertes por accidentes de motocicletas [Internet]. El Heraldo, Honduras: 2015, abril 26. Sección Sucesos. [Citado el 23 de enero de 2015]. Disponible en: http:// www.elheraldo.hn/sucesos/834743-219.

7. Bambaren Alatrista C. Características epidemiológicas y económicas de los casos de accidentes de tránsito atendidos en el Hospital Nacional Cayetano Heredia. Rev Med Hered.2004;15(1):30 - 36.

8. Higuita Y. Estudio de factibilidad para la creación de la microempresa [internet]. Medellín, Colombia: 2011 [Citado en enero de 2015]. Disponible en: http://re pository.uniminuto.edu:8080/xmlui/bits tream/handle/10656/3839/TA_Higuita John_2011.pdf?sequence=1.

9. Lascarro LG, Carrascal Villalba JM. Accidente de tránsito en motocicletas en el Municipio de Apartadó entre el 01/01/ 2006 y el 31/12/2008 [internet Tesis]. Colombia: Universidad CES; 2010[citado
14 junio 2008]. Disponible en: http://bdi gital.ces.edu.co:8080/repositorio/hand le/10946/928.

10. Peritos de Accidentes (PDA). Lesiones comunes por accidentes de motos. [internet] España: PDA;2016 [citado el 10 marzo 2016]. Disponible en: https://www. peritosdeaccidentes.com/4-lesiones-co munes-accidentes-motos.

11. Fundación Mutua Madrileña. Estudio de siniestralidad vial. [internet] Madrid:Fundación Mutua Madrileña;2013 [citado 07 octubre 2013]. Disponible en http://www. fundacionmutua.es/Detalle-Noticia.ht ml\&cid=1181564453224.

12. Donate López C. Factores de riesgo de mortalidad y morbilidad en accidentes de tráfico de ciclomotores y motocicletas [tesis doctoral]. Granada, España: Departamento de Medicina Preventiva y Salud Pública, Universidad de Granada; 2006.

13. Guzman Alonzo DP. Determinación de los factores de riesgo en accidentes donde estan involucrados motocicletas en Bogotá.[internet]; 2011 [citado 23 junio 2011].Disponible en: https://reposi tory.javeriana.edu.co/bitstream/hand le/10554/7261/tesis503.pdf?sequence $=1$.

14. Forero M. LJ, Valvuena C. LJ. Muertes y lesiones por accidente de tránsito. Colombia, 2009: eventos fatales y no fatales relacionados con el tránsito y su relación con las emociones. [internet]2009 [citado en agosto 2009], disponible en: http://www.medicinalegal.gov.co/ documents/10180/34291/5+accidentes transito.pdf/e 82bb17d-8517-4a09-a 153-ad685dacf620.

15. Veronese AM, de Oliveira DL, Shimitz Tdos S. Characterization of motorcyclist admitted in the emergency hospital of Porto Alegre. Rev Gaucha Enferm. 2006; $2(3): 9-11$. 